\title{
Interaction-induced beats of Friedel oscillations in quantum wires
}

\author{
D. F. Urban ${ }^{1}$ and A. Komnik ${ }^{1,2}$ \\ ${ }^{1}$ Physikalisches Institut, Albert-Ludwigs-Universität, \\ Hermann-Herder-Str. 3, D-79104 Freiburg, Germany \\ ${ }^{2}$ Institut für Theoretische Physik, Universität Heidelberg, \\ Philosophenweg 19, D-69120 Heidelberg, Germany
}

(Dated: October 30, 2018)

\begin{abstract}
We analyze the spectrum of electron density oscillations in an interacting one-dimensional electron system with an impurity. The system's inhomogeneity is characterized by different values of Fermi wave vectors $k_{F}=k_{L / R}$ on left/right side of the scatterer, leading to a Landauer dipole formation. We demonstrate, that while in the noninteracting system the Friedel oscillations possess only one periodicity related to the local $k_{F}$, say $k_{L}$ on the left side, the interplay of the interactions and the Landauer dipole generates an additional peak in the spectrum of density oscillations at the counterpart $k_{R}$. Being only present in correlated systems, the position and shape of this spectral feature, which in coordinate space is observable as a beating pattern in the Friedel oscillations, reveals many important details about the nature of interactions. Thus it has a potential to become an investigation tool in condensed matter physics.
\end{abstract}

PACS numbers: 72.10.Fk, 72.25.Mk, 73.63.-b

The role of electron-electron interactions is decisive in a large number of condensed matter phenomena. The correlations are not only responsible for such spectacular effects as the fractional quantum Hall effect and high$T_{c}$ superconductivity, but they also turned out to influence the physical properties of metallic $1 \mathrm{D}$ and quasi-1D materials in a very profound way. In fact, contrary to their higher dimensional relatives, which are almost perfectly described by the Fermi liquid theory, the 1D metals even constitute a special universality class of TomonagaLuttinger liquids (TLL) [1, 2]. Very naturally, it is extremely important to have precise, reliable and easily accessible methods to investigate electronic interactions. The most useful of them involve quantities which are only present in interacting systems such as, e.g., the high energy tails of field emission spectra $3,4,4,5]$.

During the last two decades scanning tunneling microscopy and atomic force microscopy as well as their descendants established themselves as some of the most efficient techniques for the investigation of low dimensional materials. It became possible to image the electronic density of, e.g., carbon nanotubes, with atomic resolution [6, 7]. In one of the recent experiments [8] even an explicit imaging of Friedel oscillations (FOs) near the end of a single-wall carbon nanotube (SWNT) has been successfully achieved, making it possible to obtain the signatures of a TLL.

The classical FO is nothing else but an electron density modulation in vicinity of a hard wall potential [9]. Due to the full reflection at a boundary at $x=0$ a coherent superposition of incoming and outgoing electron wavefunctions results in a $\sin \left(2 k_{F} x\right) / x$ behavior of the correction to the uniform electron density in the bulk (at $x \rightarrow \infty)$. The fundamental oscillation period then is defined by the Fermi wave vector $k_{F}$ and is equal to $\pi / k_{F}$. Similar density oscillations also emerge in the vicinity of any scatterer introduced into a metal. Their form and the actual particle density at the position of the scatterer of course depend on the scattering potential. One could conclude that in the case of a finite barrier transmission the modulation can change qualitatively so that a beating pattern due to two harmonic components would emerge as soon as the Fermi wave vectors on both sides of the scatterer become different, $k_{L} \neq k_{R}$. A straightforward calculation immediately shows that this is not the case as long as electron-electron interactions are not taken into account. Suppose we have a scatterer with reflection and transmission amplitudes $r$ and $t$, respectively, placed at $x=0$. Let $d_{k}$ and $a_{k}$ be the plane wave annihilation operators for the electrons injected into the system at $x \rightarrow \pm \infty$. Then the full field operator for electrons on the left side of the scatterer is

$$
\psi_{L}(x)=\int \frac{d k}{2 \pi} \nu(k)\left[\left(e^{i k x}-r e^{-i k x}\right) a_{k}+t^{*} e^{-i k x} d_{k}\right],
$$

where for simplicity we assume the band structure encoded in the density of states $\nu(k)$ to be the same on both sides of the scatterer. The particle density is then given by the average $\left\langle\psi_{L}^{\dagger}(x) \psi_{L}(x)\right\rangle$. Taking into account that cross correlations of the type $\left\langle a_{k}^{\dagger} d_{k^{\prime}}\right\rangle$ vanish, one realizes that the term proportional to $t^{*} t$ is not $x$-dependent. That is why the density oscillations on the (here) left side of the scatterer are not affected by the electrons from the right side up to an overall uniform $x$-independent shift. It is instructive to carry out the calculation for a specific system. We use the model for the conductance band of a SWNT: the electrons are assumed to be onedimensional with a dispersion relation which is the same on both sides of the scatterer and is linear $E(k)=v_{F} k$, where $v_{F}$ is the Fermi velocity (for simplicity we use units with $\hbar=e=m_{e}=1$ throughout), see Fig. 1. Different values of $k_{F}$ on both sides can be produced by applying 


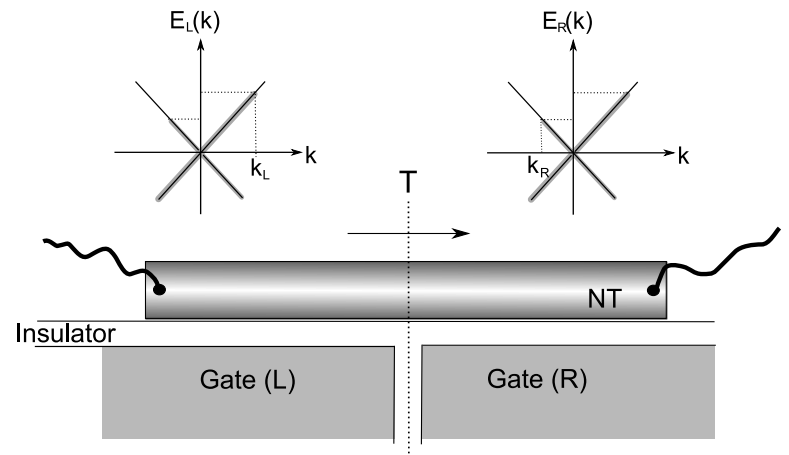

FIG. 1: Sketch of the system under investigation.

a finite bias voltage to the whole system complemented by appropriate gating of the constituent subsystems. For convenience we shall call the difference in the Fermi energies 'voltage' $V: E_{F, L / R}=v_{F} k_{L / R}=v_{F} k_{F} \pm V / 2$. Then the expectation values $\left\langle a_{k}^{\dagger} a_{k^{\prime}}\right\rangle=2 \pi \delta_{k k^{\prime}} n_{L}(k)$ and $\left\langle d_{k}^{\dagger} d_{k^{\prime}}\right\rangle=2 \pi \delta_{k k^{\prime}} n_{R}(k)$ contain the Fermi distribution functions $n_{L / R}$ with Fermi energies $E_{F, L / R}$. The particle density $\rho_{L / R}$ is given by the sum of three terms: (i) the full constant average density $\rho^{(0)}=k_{F} / \pi$; (ii) a constant shift proportional to $V$ on both sides given by

$$
\rho_{L / R}^{(V)}= \pm(1-T) V /\left(2 v_{F} \pi\right),
$$

the difference of which can be reworked into Landauer's residual resistivity dipole strength $p=k_{F} V(1-$ $T) /(2 \pi n)$, where $n$ is the density of the charge carriers [10, 11]; (iii) a space dependent oscillating part

$$
\rho_{L / R}^{(o s c)}(x)=-\frac{\sqrt{1-T}}{2 \pi} \frac{\sin \left(2 x k_{L / R}-\varphi_{r}\right)+\sin \left(\varphi_{r}\right)}{x} .
$$

Here $T=t^{*} t$ is the transmission probability and $\varphi_{r}$ is the phase shift for a reflected electron. The full constant av- erage density $\rho^{(0)}$ usually is compensated by the constant homogeneous background charge of the ions of the underlying lattice structure and must therefore be subtracted in the interaction terms. Eqs. (2) and (3) show explicitly that $\rho_{L}\left[\rho_{R}\right]$ only depends on $k_{L}\left[k_{R}\right]$ in the absence of interactions. Different ways to measure the induced chemical potential oscillations are in detail discussed in 12.

Now we want to address the effect of interactions on the FOs. We consider a spin-independent pointlike densitydensity interaction with strength $U$. In terms of the Keldysh Green's functions (GF) defined as $g_{\alpha L}^{<}(x, y, t)=$ $-i\left\langle\psi_{\alpha}(x, t) \psi_{L}(y, 0)\right\rangle$ for the lesser GF and a similar definition of the retarded GF, the first-order correction to the particle density is given by

$$
\begin{gathered}
\delta \rho_{L}(x)=4 U \operatorname{Im} \int d t \int_{-\infty}^{0} d y \\
\times \quad \sum_{\alpha=R, L} \rho_{\alpha}(y) g_{L \alpha}^{r e t}(x, \alpha y,-t) g_{\alpha L}^{<}(\alpha y, x, t),
\end{gathered}
$$

with the prescription $\alpha=R, L=( \pm)$, which accounts for two different subsystems: the left one for $y<0$ and its counterpart on the right side, $y>0$. This correction has a simple physical interpretation as it comes from the electron excursions from the measuring point $x$ to some other coordinate $y$ where it gets scattered on the local electron density $\rho_{\alpha}(y)$ (which may be on either side of the barrier) and then propagates back to the original $x$. The scattering process is crucial as it allows for exchange of momenta. In this way the information about the Fermi wave vector on the other side of the impurity is carried back to the measuring point $x$. This effect is similar to secondary tunnelling processes (Auger like scattering) responsible for high-energy tails in field emission spectra of correlated hosts $3,4,4,5]$. The GFs necessary for the evaluation of (4) are

$$
\begin{aligned}
& g_{L L}^{r e t}(x, y, t)=-i \Theta(-t) \int_{0}^{\infty} \frac{d k}{\pi} e^{-i v_{F} k t}\left\{\cos [k(x-y)]-\sqrt{1-T} \sin \left[k(x+y)-\varphi_{r}\right]\right\} \\
& g_{L L}^{<}(y, x, t)=-\int_{0}^{\infty} \frac{d k}{2 \pi i}\left\{n_{L} e^{i k(y-x)}+\left[n_{L}(1-T)+n_{R} T\right] e^{-i k(y-x)}-2 n_{L} \sqrt{1-T} \cos \left[k(y+x)-\varphi_{r}\right]\right\} e^{-i v_{F} k t}, \\
& g_{L R}^{r e t}(x, y, t)=-i \Theta(-t) \frac{\sqrt{T}}{\pi} \int_{0}^{\infty} d k e^{-i v_{F} k t} \sin \left[k(x+y)-\varphi_{r}\right], \\
& g_{R L}^{<}(y, x, t)=-\int_{0}^{\infty} \frac{d k}{2 \pi}\left\{n_{L} \sqrt{T} e^{-i k(y+x)+i \varphi_{r}}-n_{R} \sqrt{T} e^{i k(y+x)-i \varphi_{r}}-\sqrt{T(1-T)}\left(n_{L}-n_{R}\right) e^{i k(x-y)}\right\} e^{-i v_{F} k t},
\end{aligned}
$$

where $\Theta$ denotes the unit step function. Note that these GFs are exact in scattering strength since we are using the exact scattering states. As we are looking for the secondary FO (contrary to the primary one coming from the 
native $k_{L}$ ) it is more convenient to perform the Fourier transform of the correction to the density. Furthermore, we would like to concentrate on the consequences of having two different Fermi levels in the system, thus we only keep terms proportional to the difference of the Fermi distribution functions $\Delta n=\left(n_{L}-n_{R}\right)$.

The detailed evaluation of (44) reveals two contributions to the first order density correction $\delta \rho$. The first one comes from the mean shift $\rho_{L / R}^{(V)}$ of the zeroth order density due to a finite $V$ and it is found to be given by

$$
\begin{aligned}
& \delta \widetilde{\rho}_{L}^{(V)}(p)=\frac{T(1-T) V U}{4\left(\pi v_{F}\right)^{2}} \\
& \quad \times \sum_{\xi= \pm} \frac{1+e^{-2 i \xi \varphi_{r}}}{\xi p}\left\{\pi \Theta\left(\xi p+2 k_{L}\right) \Theta\left(-\xi p-2 k_{R}\right)\right. \\
& \left.\quad-i \xi \sum_{\alpha=R, L} \alpha \log \left|1+\frac{2 k_{\alpha}}{\xi p}\right|\left[1-\Theta\left(\xi p+k_{\alpha}\right)\left(1+e^{2 i \xi \varphi_{r}}\right)\right]\right\}
\end{aligned}
$$

The spectrum of the density profile shows up two distinct peaks at $p= \pm 2 k_{L / R}$ while in the noninteracting case there would be only one native periodicity with wavelength $\pi / k_{L}$. The peaks are in fact logarithmic singularities due to the one-dimensional geometry of the system. By construction the signal strength is proportional to the applied voltage. It vanishes for both perfect transmission, when translational invariance of the system is restored and no buildup of FOs is possible, and perfect reflection, when the electrons on the one side of the barrier cannot acquire information about the Fermi edge on the other side. The prefactor $T(1-T)$ can indeed also be explained more intuitively. Suppose we have a wave packet approaching the barrier coming from the left reservoir. At the boundary a part of it is reflected with an amplitude $\sim \sqrt{1-T}$. The transmitted part which is $\sim \sqrt{T}$ propagates to the point $y$ where it interacts with the wave packet coming from the right reservoir, which on its way to $y$ is reflected from the boundary picking up a prefactor $\sim \sqrt{1-T}$. The interaction process yields a prefactor $U$ and the propagation back through the barrier gives another $\sqrt{T}$, see Fig. 2. Thus the full prefactor is $(\sqrt{T} \sqrt{1-T})^{2}=T(1-T)$. For a representative set of parameters the density spectra as given by (5) are plotted in the left panels of Fig. 3. Being transformed back into the coordinate space, the two peaks result in a beating pattern of the density oscillation as a function of distance from the impurity.

Besides these oscillations, originating from the finite (average) coordinate-independent difference in chemical potentials, there is, of course, a contribution from the oscillating part of the density on the other side of the barrier. The physical motivation and qualitative dependence on the transmission is the same as discussed above, but corresponding expressions are rather long, so we restrict ourselves to the plots shown in the right panels of Fig. 3. As before, distinct features arise around both dou-

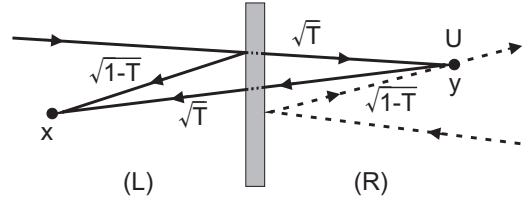

FIG. 2: Graphical representation of the processes contributing to the secondary Friedel oscillations, see text.

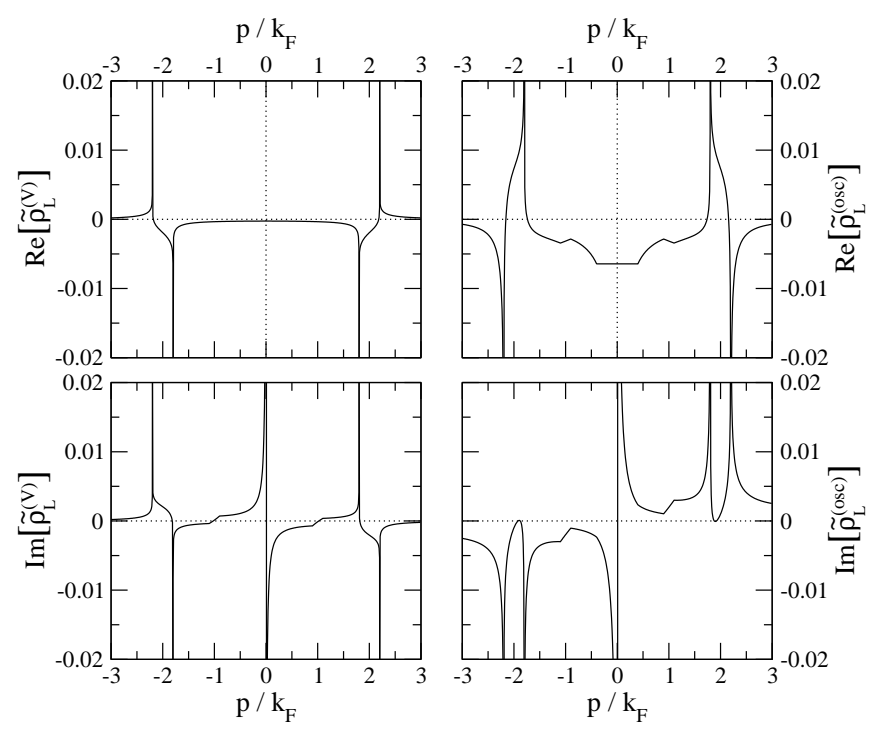

FIG. 3: Real and imaginary parts of the density oscillations spectrum (arbitrary units) for $V=0.1 k_{F}^{-1}$. Left panels: contributions from $\rho_{\alpha}^{(V)}$. Right panels: contribution from the oscillating part of the density $\rho_{\alpha}^{(o s c)}$.

bled Fermi momenta $p= \pm 2 k_{L / R}$. We expect that this interaction signature should be observable in dedicated experiments, thereby giving important insights into the nature of electronic correlations.

So far we treated the system exactly in the tunnelling (scattering) amplitude and performed a weak interaction expansion. In strictly $1 \mathrm{D}$ environments, at least in equilibrium, the electron system belongs to the universality class of TLLs. In this case the interactions have to be treated nonperturbatively. For the FOs at an open boundary (at a hard wall) the density profile can be calculated analytically and numerically for arbitrary interaction strength [13, 14, 15]. In our case the situation is much more difficult as we must allow for at least weak electron tunnelling at a barrier between two half-infinite TLLs. It turns out, that such a situation indeed describes the low-energy fixed point of a TLL with an impurity [16, 17]. Therefore we adopt this model and perform a calculation which is 'dual' to the one presented above, taking into account the interactions exactly while performing a perturbation expansion in the tunnelling amplitude.

The beating pattern in the density oscillations already 
shows up in the inhomogeneous case (which is more convenient to handle), when a noninteracting subsystem is coupled to a genuine TLL and the FOs are measured in the interacting part The correction to the density profile is then found to be given by

$$
\delta \rho(x)=\sum_{\zeta= \pm} e^{i \zeta\left(2 k_{F}+V / v_{F}\right) x} \delta\left\langle\psi_{L}^{\dagger}(-\zeta x) \psi_{L}(\zeta x)\right\rangle .
$$

The evaluation of the expectation value on the rhs is accomplished with the help of the bosonization representation of the TLL Hamiltonian with the interaction of the kind we already have used in the first part of this Letter. The correlation strength translates into the dimensionless interaction parameter $g=1 / \sqrt{1+U /\left(\pi v_{F}\right)}$, with $0<g<1$ for repulsive interaction. The correction to the density is

$\delta\left\langle\psi_{L}^{\dagger}(x) \psi_{L}(-x)\right\rangle=\frac{2 \gamma^{2}}{\pi v_{F}} \frac{a_{0}^{g+1 / g}}{\left(\pi a_{0}\right)^{2}|2 x|^{g}} e^{i(\pi / 2) \operatorname{sign}(x)} F(x)$,

where $a_{0}$ is the lattice spacing of the underlying lattice model. $F(x)$ is an oscillatory function, whose Fourier transform can be written in the form

$$
\begin{aligned}
\widetilde{F}(k) & =\operatorname{Re}\left\{-\int_{0}^{\infty} d \tau_{+} \int_{-\tau_{+}}^{\tau_{+}} d \tau_{-}\left(\frac{-i}{\tau_{-}+i \delta}\right)^{1 / g}\right. \\
& \times \frac{\tau_{+}+\tau_{-} / 2}{2 \tau_{+}+i \delta} e^{i k \tau_{+}+i\left(k / 2+V / v_{F}\right) \tau_{-}} \\
& -\sum_{\xi= \pm} \xi \int_{0}^{\infty} d \tau_{+} \int_{0}^{\tau_{+}} d \tau_{-}\left(\frac{\xi i}{\tau_{-}-i \xi \delta}\right)^{1 / g} \\
& \left.\times \frac{\tau_{+}+\xi \tau_{-} / 2}{2 \tau_{+}+i \delta} e^{i \xi k \tau_{+}+i\left(k / 2+V / v_{F}\right) \tau_{-}}\right\}
\end{aligned}
$$

As $1 / g>1$ for repulsive interactions it is the $\tau_{--}$ dependent parts in the brackets which dominate the behaviour of $\widetilde{F}(k)$. A stationary phase argument with respect to the $\tau_{-}$integration yields the condition $k / 2+$ $V / v_{F}=0$ for the maximum of $\widetilde{F}(k)$. This is confirmed by the numerical evaluation of (7) shown in Fig. 4, where $\widetilde{F}(k)$ is plotted for different values of the interaction parameter $g$. Therefore we conclude that the correction (6) shows up oscillations with period $\pi / k_{R}$ in addition to the native one with $\pi / k_{L}$ just like in the perturbative interaction calculation. Interestingly, the stronger the interactions the more pronounced are these newly arising oscillations. Similar effects in a slightly different setup have been reported in Ref. [18].

To conclude, we have discussed the density profile of Friedel oscillations in a 1D metal with different Fermi wave vectors on either side of an imbedded impurity. Contrary to the naive expectation in the absence of interactions, the density oscillations possess only one periodicity originating from the local value of $k_{F}$ even when the impurity has a finite transmission coefficient. The situation changes dramatically in interacting systems, where

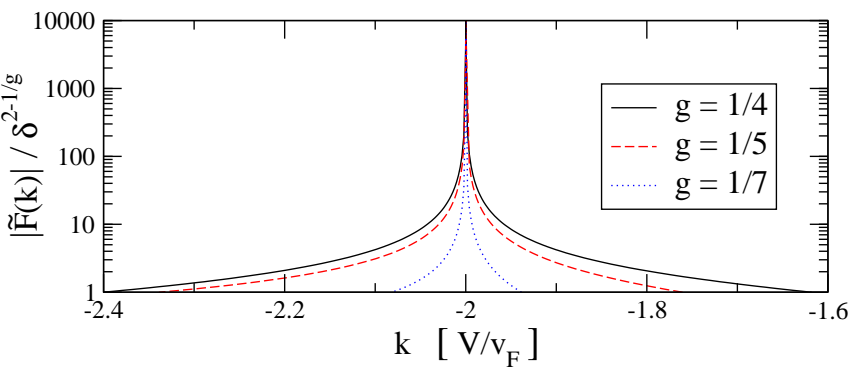

FIG. 4: The function $\widetilde{F}(k)$ as given by Eq. (7) plotted for different values of the interaction parameter $g$.

the spectral function acquires an additional peak, which is a result of the interplay of interactions and the Landauer dipole. We have shown this in two different situations by first taking into account interactions perturbatively and using the exact scattering states. In a second step we have considered the dual situation of a weak link between two TLLs where the electronic correlations have been taken into account with the help of bosonization technique. We are convinced that the very fact, that the beating pattern owes its existence to the interactions has the potential to make measurements of Friedel oscillations (which by now have become unproblematic) an important tool to study electronic correlations in 1D metals and carbon nanotubes in future experiments.

The authors would like to thank H. Grabert and R. Egger for many interesting discussions. A.K. is supported by the DFG grant No. KO 2235/2 (Germany).

[1] F. Haldane, J. Phys. C 14, 2585 (1981).

[2] J. Luttinger, J. Math. Phys. 4, 1154 (1963).

[3] C. Lea and R. Gomer, Phys. Rev. Lett. 25, 804 (1970).

[4] J. Gadzuk and E. Plummer, Rev. Mod. Phys. 45, 487 (1973).

[5] A. O. Gogolin and A. Komnik, Phys. Rev. Lett. 87, 256806 (2001).

[6] T. Odom, J.-L. Huang, P. Kim, and C. Lieber, Nature 391, 62 (1998).

[7] L. C. Venema, V. Meunier, P. Lambin, and C. Dekker, Phys. Rev. B 61, 2991 (2000).

[8] J. Lee, S. Eggert, H. Kim, S.-J. Kahng, H. Shinohara, and Y. Kuk, Phys. Rev. Lett. 93, 166403 (2004).

[9] G. Mahan, Many-Particle Physics (Plenum, New York, 1990).

[10] W. Zwerger, L. Bönig, and K. Schönhammer, Phys. Rev. B 43, 6434 (1991).

[11] R. Landauer, Z. Physik B 21, 247 (1975).

[12] M. Büttiker, Phys. Rev. B 40, 3409 (1989).

[13] R. Egger and H. Grabert, Phys. Rev. Lett. 75, 3505 (1995).

[14] M. Fabrizio and A. O. Gogolin, Phys. Rev. B 51, 17827 (1995).

[15] A. Leclair, F. Lesage, and H. Saleur, Phys. Rev. B 54, 
13597 (1996).

[16] C. L. Kane and M. P. A. Fisher, Phys. Rev. B 46, 15233 (1992).

[17] A. Furusaki and N. Nagaosa, Phys. Rev. B 47, 4631
(1993).

[18] R. Egger and H. Grabert, Phys. Rev. Lett. 77, 538 (1996). 